\title{
Gestión de la salud pública y políticas públicas para estimular estilos de vida saludables a nivel local y global
}

\author{
Management of public health and public policy to encourage healthy lifestyles at local and \\ global level
}

Jarentzy Cruz Olvera ${ }^{a}$, Verónica Mendieta Ramírez ${ }^{a}$

\begin{abstract}
:
The management and participation of each of Mexicans in regard to maintaining balance in lifestyle and health, constitutes rights and obligations for the binomial government-citizen, that is to say, from responsibility, both with the obligation to do for the health, so the aim of this trial was a venture into the generation of knowledge about health management from a literature review with emphasis on the essential functions of public health, to propose strategies aimed at improving in styles and quality of life, hence the health status of the population. Government institutions require stimulate by managing quality of health services in the coresponsibility efforts with community participation with emphasis on transforming lifestyles, so that people with poor lifestyle represent itself a shared social challenge.
\end{abstract}

Keywords:

management, participation, responsibility, quality, lifestyles.

\section{Resumen:}

La gestión y participación de cada uno de los mexicanos en lo que respecta a mantener el equilibrio en estilo de vida y salud, se constituye en derechos y obligaciones para el binomio gobierno-ciudadano, es decir, desde la corresponsabilidad, ambos con la obligación de hacer por la salud, por lo que el objetivo del presente ensayo se centró en incursionar en la generación de conocimiento respecto a gestión en salud a partir de una revisión bibliográfica con énfasis en las funciones esenciales de la salud pública, para proponer estrategias orientadas a mejorar en estilos y calidad de vida, y de ahí en el estado de salud de la población. Las instituciones de gobierno requieren estimular mediante la gestión de calidad de sus servicios de salud, en corresponsabilidad del esfuerzo compartido con la participación comunitaria con énfasis en la transformación de los estilos de vida, por lo que, personas con estilo de vida deficiente representan en sí un reto social compartido.

\section{Palabras Clave:}

Gestión, participación, corresponsabilidad, calidad, estilos de vida.

\section{Introducción}

La Gestión en Salud se define como la aplicación de la administración de bienes y servicios, mediante la planeación estratégica participativa que genere una mejora continua en el bienestar del ser humano que se traduce en salud. Es importante diferenciar "Administración" de "Gestión en Salud", la primera se refiere a la organización de recursos para lograr los objetivos planteados mediante buenos procesos y la segunda: la aplicación de estrategias efectivas dirigidas a mejorar continuamente el bienestar y las condiciones de salud en un determinado contexto. La gestión de salud involucra de manera directa al profesional de la salud, que funciona como gestor de la misma, al hacer buen uso de los recursos disponibles considerando la planeación y que mediante los procesos adecuados, optimizando los procesos y con ello eficientice la accesibilidad a los servicios de salud; un buen gestor tomará con perspicacia el desarrollo de sus objetivos y estará al pendiente del desarrollo profesional de todos los miembros que pertenezcan a su área de especialización en su ámbito laboral con base a los siguientes objetivos. 
Reformar la normativa institucional con la visión de mejorar al interior e irradiar esa mejora donde se requiera impulsar la calidad.

Consolidar un sistema de gestión de la calidad que atienda eficazmente el desarrollo y los problemas estructurales de la gestión en concordancia con el Plan de Desarrollo que conlleven a hacer más eficientes los procesos de calidad desde el ámbito local e impactar positivamente a nivel nacional.

Profesionalizar a los actores de generar políticas públicas contando con personal que cubra sus puestos administrativos de acuerdo a las necesidades sociales.

Desarrollar acciones para mejorar el nivel de implementación de la carrera administrativa e incentivar el desarrollo del personal administrativo. Contar con recursos humanos, materiales y financieros, donde se incluya no solo de instalaciones e infraestructura tecnológica que den respuesta efectiva y eficiente a las necesidades de la población.

La gestión de calidad implica implementar cambios en aspectos tan importantes como son los hábitos de alimentación cotidiana e incluso de actividad física.

La calidad representa un imperativo ético en salud, donde existen compromisos sociales con la población humana y esta es corresponsable a su vez de auto gestionarse a sí mismo para hacer las cosas bien en cuanto a sus cuidados en su salud. La calidad es algo muy sencillo; implica hacer las cosas bien, para su desarrollo, consiste en contar con personas dispuestas a prestar un buen servicio, comprometido socialmente, con ética y valores como la responsabilidad.

En los procesos de gestión de la calidad se encuentra inmerso el análisis y estandarización de lo que hacemos, al re-direccionar y tomar decisiones en el plano administrativo, desde la organización y planeación de las estrategias de intervención, fomentando así, la probabilidad de éxito.

La gestión en salud implica, tomar en cuenta la relación costo-beneficio, que al optimizar los procesos, disminuye los costos, aumenta las beneficios, que serán visibles en la calidad de vida y la salud de la población.

\section{Se requiere entonces, de gerentes de la salud pública con compromiso social muy claro, que tengan sobre todo un fuerte compromiso social, académico y científico respecto al logro de los resultados esperados.}

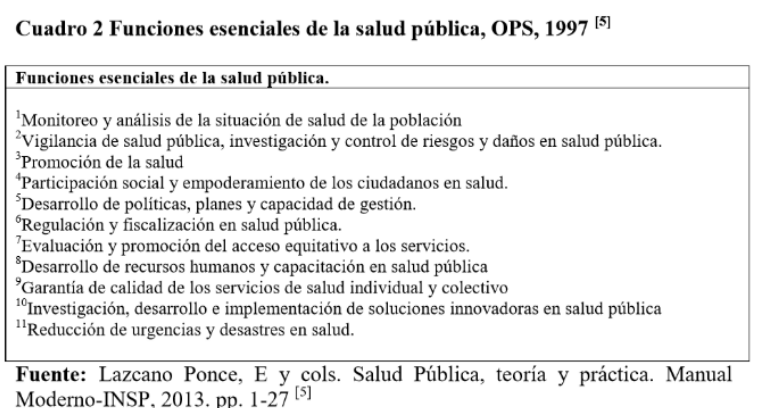

\section{Discusión}

La consideración de los determinantes de la salud como la biología humana, estilos y calidad de vida, ambientales y la asistencia sanitaria es indispensable en el diseño de estrategias de educación en salud pública desde el enfoque constructivista para generar la construcción del conocimiento, aprender a vivir mejor, el mejoramiento continuo del propio estado de salud considerando que la gestión y la calidad en salud se automotivan mediante la corresponsabilidad.

La reforma del Sistema de Salud Mexicano pretende disminuir la pobreza, la inequidad, la inaccesibilidad y las desigualdades en la atención de la salud mediante el Seguro Popular.

Respecto a gestión de servicios de salud, en específico se retoma desde las mismas funciones esenciales de la salud pública (OPS, 1997) en la quinta función denominada "Desarrollo de políticas y capacidad de gestión, que respecto al bien público señala "Contar con políticas públicas saludables que se traducen en normas y programas para desarrollar estilos de vida saludables, elección de opciones más saludables promoviendo las condiciones de accesibilidad universal a las mismas." $Y$ entre sus acciones y estrategias alude a

1. Políticas de salud pública coherentes con el contexto político y económico.

2. Planificación estratégica nacional y apoyo a la planificación en los niveles subnacionales

3. Definición y perfeccionamiento de los objetivos de la salud pública.

4. Desarrollo de códigos y leyes que guíen la práctica de la salud pública.

5. Desarrollo y fiscalización de normas dirigidas al control de riesgos de salud ambiental

6. Definición de objetivos y prioridades del sistema nacional de salud.

7. Diseño e implementación de programas para salud poblacional y

8. Competencias para la toma de decisiones basadas en pruebas científicas.

Entre las otras funciones esenciales de la salud pública existe "Monitoreo y análisis de la situación de salud de la 
población" motiva a generar cambios importantes respecto a gestión al referirse en lo que respecta al bien común a:

- Contar con un sistema estadístico.

- Contar con información que permita evaluar las acciones de la salud pública.

- Contar con un sistema que permita detectar, vigilar, evaluar urgencias de salud pública.

- Acceso a la información.

La Constitución Mexicana en el artículo $1^{\circ}$ imprime, todas las personas gozaran de los derechos humanos reconocidos en la Constitución así como en los tratados Internacionales, así mismo en el artículo $4^{\circ}$ en los siguientes términos: "Toda persona tiene derecho a la protección de la salud".

Otro concepto fundamental respecto a la gestión de los servicios de salud es el propuesto como "Epidemiología de gestión" que se refiere al uso de los métodos epidemiológicos en la programación y evaluación de servicios de salud.

La salud pública juega un rol fundamental tanto en la identificación de factores determinantes y sus posibles soluciones como el cambio en el estilo de vida, la implementación de medidas poblacionales para su control y la evaluación de su eficacia.

Es evidente la responsabilidad del gobierno como impulsor de la creación de un mejor modelo de atención, son insuficientes las instalaciones y los grupos que trabajan en investigación, prevención y tratamiento de la obesidad.

Se debe enfatizar en los beneficios de las conductas, educar a los pacientes sobre las expectativas del tratamiento, y discutir los riesgos y beneficios del modelo, son estrategias que se pueden utilizar para iniciar un nuevo estilo de vida.

Un problema actual de salud pública en México es la obesidad, nuestro país ocupa el primer lugar de prevalencia en el mundo, y es la punta de lanza para que se desarrollen estas enfermedades, por lo que es uno de los principales retos en nuestro sistema de salud, pero, mientras no se modifique el estilo de vida no se resolverá de base este grave problema.

Un objetivo, es la participación activa de la comunidad y el fomento de actividades positivas en lo que respecta a salud, ello entraña el reconocimiento del carácter complementario de la educación del individuo y de la comunidad, para que participe efectivamente en las actividades de salud y desarrollo.

Se deberá considerar en una perspectiva intersectorial en la que los objetivos de salud sean parte integrante de la labor de la comunidad por mejorar la calidad de vida. Los administradores de salud deben extender sus servicios a la comunidad, atender situaciones de riesgo en virtud del medio ambiente, estilo de vida, los servicios de salud, la herencia (biológica) y a los propios agentes biológicos, químicos, físicos, entre otros.

\section{Comentario Personal}

La administración en lo que se refiere a Gestión en Salud es una parte muy importante para la prestación de servicios a la población, en las que la Salud Publica tiene un papel preponderante para la planeación de programas que ofrezcan resolver las condiciones que la población demanda en base a los diagnósticos situacionales de cada región y que si no fuera por la Salud Publica, no se tendrían los elementos suficientes como para poder demostrar y planificar los recursos que se utilizan en los diferentes programas según las necesidades de una comunidad. Con la visión del cumplimiento como principales objetivos mejorar y erradicar aquellas barreras tanto sociales como económicas en un momento dado ambientales, que interfieran en el desarrollo de una comunidad. Considerar siempre como principios básicos la optimización de recursos que garanticen una eficiencia en la accesibilidad a los servicios de salud con una participación y corresponsabilidad de la comunidad; desarrollando las acciones para mejorar los recursos tanto materiales, financieros y humanos que sean necesarios. Lo anterior, tendrá que ser con hincapié en una Gestión de Calidad en donde la toma de decisiones sea la mejor para garantizar como producto final el cambio de hábitos y mejoramiento de los estilos de vida, con una actitud constructivista, así como fuerte compromiso en lo social, lo académico y lo científico, sin olvidar el sentido humano que conlleva el resolver las necesidades mínimas e indispensables para restablecer el grado de salud de una población; de esta manera se lograra elevar la esperanza de vida, sobre todo para aquellos pueblos aun marginados en donde las condiciones ambientales, biológicas, estilos de vida y sobre todo asistencia sanitaria aún siguen manifestándose desfavorables, en donde el nivel de educación será una de las principales estrategias para generar un cambio que junto con la Gestión y la adecuada optimización podremos disminuir niveles de pobreza, inequidad, inaccesibilidad, y desigualdades en la atención de salud.

Lo antes mencionado se encuentra contenido en una de las principales funciones esenciales de la Salud Publica, que es el desarrollo de políticas y capacidad de Gestión que se traducen en normas y programas para desarrollar estilos de vida saludables, promoviendo las condiciones de accesibilidad universal a las mismas y q a su vez se favorece uno de los principios de la atención primaria

a

la salud. 
Cabe señalar que al eliminar las practicas burocráticas innecesarias, se garantizaría la adecuada adquisición y el destino final de los recursos para los fines con los cuales han sido creados originalmente y que mediante la buena administración pública de los mismos se eliminaran inequidades y desigualdades en aquellos grupos vulnerables que tanto necesitan de dichos recursos y que mediante la adecuada formación de las nuevas generaciones de los profesionales de la salud, será uno de los cambios que generen grandes beneficios en los diferentes modelos de atención con los cuales cuenta el sistema Nacional de Salud.

Resulta evidente la gran responsabilidad del gobierno para el mejoramiento de los modelos de atención con los cuales ya se cuenta y que al favorecer las buenas conductas se garantizara la correcta Gestión de los Recursos de Salud.

\section{Conclusión}

Es importante por lo tanto que se trabaje en estilos y calidad de vida, en modificar indicadores de salud de la población, en tomar en cuenta el aspecto no solo económico, sino, el aspecto humanitario para buscar incrementar la calidad del estado de salud de las personas.

\section{Referencias}

[1] Ruvalcaba Ledezma J. C., Gestión de la salud pública y políticas públicas para estimular estilos de vida saludables a nivel local y global. KASMERA 2015; 43(1): 234-50. 